\title{
Incidence of Extended-Spectrum
} Beta-Lactamase-Producing Klebsiella pneumoniae among Patients and in the Environment of Hassan II Hospital, Settat, Morocco

\author{
Samira Natoubi1,2*, Abouddihaj Barguigua3,4, Sanaa Bouhali Zriouil4, Nezha Baghdad1, \\ Mohammed Timinouni ${ }^{3}$, Abderraouf Hilali'2 ${ }^{2}$ Souad Amghar ${ }^{5}$, Khalid Zerouali ${ }^{4,6}$ \\ ${ }^{1}$ Microbiology Laboratory, Hassan II Hospital, Settat, Morocco \\ ${ }^{2}$ Agrofood and Health Laboratory, Faculty of Science and Technology, University Hassan I, Settat, Morocco \\ ${ }^{3}$ Molecular Bacteriology Laboratory, Pasteur Institute of Morocco, 1, Place Louis Pasteur, Casablanca, Morocco \\ ${ }^{4}$ Microbiology Laboratory, University Hospital Center, Ibn Rochd, 1 Street Hospital, Casablanca, Morocco \\ ${ }^{5}$ Laboratory of Improved Soil Productivity and Environment, University Mohammed V, Higher Normal School, \\ Rabat, Morocco \\ ${ }^{6}$ Faculty of Medicine and Pharmacy, University Hassan II, Casablanca, Morocco \\ Email: "samiranatoubi@yahoo.fr
}

Received 1 February 2016; accepted 8 March 2016; published 11 March 2016

Copyright (C) 2016 by authors and Scientific Research Publishing Inc.

This work is licensed under the Creative Commons Attribution International License (CC BY).

http://creativecommons.org/licenses/by/4.0/

(c) (i) Open Access

\section{Abstract}

Aim: The aim of the current study is to determine: (1) the prevalence of extended-spectrum $\beta$-lactamase-producing $K$. pneumoniae (ESBL-Kp) isolated from clinical samples and a hospital environment in Hassan II Hospital (Settat, Morocco); (2) the associated risk factors of ESBL-Kp infections; (3) the link between clinical and environmental isolates. Methods: During the study period (April 2010 to March 2011), all patients infected and hospital environment sites contaminated by $K$. pneumoniae were considered as the potential study population and environmental site. The clinical data were collected to identify risk factors for ESBL carriage of $K$. pneumoniae infection. Screening of ESBL-and carbapenemase-producing isolates was performed by using a double-disk synergy test and the modified Hodge test, respectively. ESBL-Kp isolates were tested for the presence of genes encoding $\beta$-lactamases and were investigated by PCR. The clonal relationship between

${ }^{*}$ Corresponding author.

How to cite this paper: Natoubi, S., et al. (2016) Incidence of Extended-Spectrum Beta-Lactamase-Producing Klebsiella pneumoniae among Patients and in the Environment of Hassan II Hospital, Settat, Morocco. Advances in Microbiology, 6, 152-161. http://dx.doi.org/10.4236/aim.2016.63015 
ESBL-producing isolates was analysed by ERIC- and REP-PCR method. Results: The overall prevalence of ESBL-Kp among clinical and environmental $K$. pneumoniae isolates was $35.13 \%(13 / 37)$ and $4.04 \%(4 / 99)$, respectively. The main risk factors for carrying ESBL-Kp were renal disease $(46.15 \%)$, recent surgery $(53.84 \%)$, previous hospitalisation $(76.92 \%)$, and the presence of many invasive devices $(53.84 \%)$. All ESBL isolates were multidrug resistant. The bla $a_{\text {CTX-м }}$ group1and $b^{b l a} a_{\text {SHV }}\left(70.58 \%\right.$ for each) were the most prevalent followed by $b a_{\text {TЕм }}(52.94 \%)$. Thirteen strains expressed at least two bla genes. One isolate was positive in the modified Hodge test and was a bla $_{\text {OXA-48 }}$ producer. ERIC and Rep-PCR methods revealed an epidemic clonal dissemination of these isolates. Conclusion: The emergence of OXA-48 carbapenemase, endemic clonal dissemination and multi-drug resistance of ESBL-Kp isolates in our institution is highly alarming.

\section{Keywords}

ESBL-Producing K. pneumoniae Infections, Hospital Environment, Risk Factors

\section{Introduction}

Klebsiella pneumoniae is an important pathogen known to cause nosocomial infections, such as urinary tract as well as respiratory tract infections and septicemia. Moreover, the infections caused by this isolate can be extended by the hospital environment. K. pneumoniae persists to be the major ESBL-producing organism isolated in the hospital settings all over the world. Infections due to ESBL-producing K. pneumoniae (ESBL-Kp) increased mortality in hospitalised patients [1]. Risk factors for the acquisition of ESBL-Kp involve mechanical ventilation, the length of hospital stay especially in the intensive care units ICU, and previous use of antibiotics, identified to be the most prevalent risk factors for ESBL-producing bacteria [2]. Various class of $\beta$-lactamases have been described in ESBL-Kp, within the class A ESBL types, including the TEM, SHV and CTX-M, is the most extensive and clinically relevant [3]. The ESBL are commonly plasmid encoded. The Plasmid usually carry genes encoding resistance to other antimicrobial agents, it is frequently responsible for ESBL production [4]-[6]. In Moroccan hospitals, the existence of ESBL-producing organisms has been reported and their prevalence varies from $8 \%$ to 51\% [7] [8]. Nevertheless, various epidemiological factors combined with ESBL-producing strains acquire to be described. Nosocomial infections caused by K. pneumoniae may lead to a potentially lifethreatening condition [2] [9]. Knowledge of local epidemiology is required to define the practical treatment for patients with a hospital infection.

The present study aims to determine the rate of ESBL-Kp isolated from clinical samples and hospital environment sites, and to find the relation between them. We have also determined the resistance of ESBL strains to other antimicrobial groups and analysed risk factors associated with the rate of infections by ESBL-producing strains.

\section{Materials and Methods}

\subsection{Design, Setting and Recruitment}

Patients’ recruitment took place between April 2010 and March 2011 at Hassan II Hospital. This hospital is situated in Settat city (72 km on the north side of Atlantic Casablanca, Morocco). The hospital structure is composed of five major departments (emergency and intensive care, mother child, surgery, medicine and medicotechnical) with a capacity of 302 beds. The Hassan II Hospital is a referral hospital for a population of approximately 1 million in the Settat area.

In this study, patients admitted to Hassan II Hospital were required to have nosocomial K. pneumoniae isolates coming from clinical specimens during their hospital stay. According to the Center for Disease Control (CDC) criteria, the diagnosis of nosocomial infection was established. Previous hospitalisation was defined as an admission at Hassan II Hospital or another hospital within 30 days prior to the current admission. The origin of the isolate was accepted as nosocomial if the strain was isolated more than 48 hours after hospitalisation.

Microbiological specimens were collected when the attending physician suspected infection based on systemic 
signs (unexplained fever, chills and hypotension) and/or local signs (purulent tracheal aspirates in mechanically ventilated patients, purulent urinary drainage, or pus or pain at a vascular catheter insertion site). Microbiological specimens were collected as recommended by the CDC.

\subsection{Data Collection}

Data involve basic demographic characteristics (age, sex, pre-infection hospital stay, and nosocomial origin), in addition to the comorbid diseases (surgical intervention; renal, respiratory and central nervous diseases; along with many more). The data involve also intensive care unit admission, previous hospitalisation, recent surgery, and duration of hospital stay.

\subsection{Environmental Samples}

Environment samples were collected from the equipment and surfaces of the medical, intensive care unit (ICU), surgery, paediatric and maternity wards. Sampling of contaminated surface can be done with cotton-tipped swabs (Copan Diagnostics, California, USA), moistened with nutrient broth before use then placed in $1.5 \mathrm{~mL}$ of the same solution. Samples were then vortexed before being incubated overnight at $35^{\circ} \mathrm{C}$.

\subsection{Culture Methods}

After being incubated overnight, the nutrient broth samples were recorded to show either growth or no growth, based on the presence of a turbid solution. The samples showing growth were then plated onto Mac Conkey (MAC) agar and incubated overnight at $35^{\circ} \mathrm{C}$. Any colonies on MAC agar were identified using conventional methods, as well as the commercial identification kit API 20E (Biomerieux, Marcy l'Etoile, France).

Antimicrobial drug susceptibility testing for $K$. pneumoniae isolates was performed according to the disk diffusion method on Mueller-Hinton agar medium, and the results were interpreted according to the recommendations by the Clinical and Laboratory Standards Institute (CLSI) [10]. The following antimicrobial agents (BioRad, Marnes-la-Coquette, France) were tested: amoxicillin/clavulanic acid (20/10 $\mu \mathrm{g})$, cephalothin (30 $\mu \mathrm{g})$, cefoxitin (30 $\mu \mathrm{g})$, cefotaxime (30 $\mu \mathrm{g})$, ceftazidime (30 $\mu \mathrm{g})$, aztreonam (30 $\mu \mathrm{g})$, ciprofloxacin (5 $\mu \mathrm{g})$, gentamicin (10 $\mu \mathrm{g})$, amikacin (30 $\mu \mathrm{g})$, trimethoprim/sulfamethoxazole (1.25/23.75 $\mu \mathrm{g})$, imipenem (10 $\mu \mathrm{g})$ and ertapenem $(10 \mu \mathrm{g})$. ESBL production was screened using a double disk with a synergy test between third-generation cephalosporin (cefotaxime, ceftazidime) or a monobactam (aztreonam) disks, placed at a distance of $30 \mathrm{~mm}$ (centre to centre) from the amoxicillin/clavulanic disk as previously described [11]. This test is considered as positive if we noted a zone of inhibition between the cephalosporin antibiotics and amoxicillin/clavulanic disk, after 24-h incubation.

The detection of ESBL among AmpC hyper-producers was assessed by a double combination disk test on cloxacillin $(250 \mu \mathrm{g} / \mathrm{mL})$ agar [11]. The standard strains E. coli ATCC 25922 and K. pneumoniae ATCC 700603 were used as a negative and a positive control of ESBL production. All the isolates with a reduced susceptibility to an imipenem disk (diameter of zones of inhibition, $<22 \mathrm{~mm}$ ) or an ertapenem disk (diameter of zones of inhibition, $\leq 21 \mathrm{~mm}$ ) were screened for the production of carbapenemase by using the modified Hodge test according to the recommendations of the CLSI [10].

\subsection{Molecular Studies}

\subsubsection{Preparation of DNA Template for PCR}

Total DNA was extracted by the boiling method by suspending a few colonies of overnight culture of $\mathrm{K}$. pneumoniae isolates growing on Luria Bertani agar (Bio-Rad) in $500 \mu \mathrm{L}$ of DNase-and RNase-free water (Invitrogen, Paisley, UK). The suspension was boiled at $100^{\circ} \mathrm{C}$ for 10 minutes in a thermal block (Polystat 5, Bioblock Scientific, France), then centrifuged at $19,000 \mathrm{x}$ g for 5 minutes. An aliquot of $1 \mu \mathrm{L}$ of the supernatant was used as the DNA template for PCR.

\subsubsection{Detection of $\beta$-Lactamase-Encoding Genes}

K. pneumoniae isolates were screened by PCR for the following $\beta$-lactamase-encoding genes: bla $a_{\mathrm{CTX}-\mathrm{M}}$ group 1 , bla $a_{\mathrm{CTX}-\mathrm{M}}$ group 9, bla $a_{\mathrm{CTX}-\mathrm{M}}$ group 2, bla $a_{\mathrm{TEM}}, b l a_{\mathrm{SHV}}, b l a_{\mathrm{OXA}-48}, b l a_{\mathrm{NDM}}$, bla $a_{\mathrm{KPC}}$ and $b l a_{\mathrm{VIM}}$ as described previously [12]. The known $\beta$-lactamase-producing strains E. coli U2A1790 (CTX-M-1), Salmonella spp. U2A1446 (TEM-1 
and SHV-12), E. cloacae U2A 2242 (KPC-3) and K. pneumoniae U2A 2016 (VIM-1) were used as positive controls. The $E$. coli $\mathrm{K}_{12} \mathrm{~J}_{5}$ strain was used as a negative control. PCR products were detected by electrophoresis on a $1.5 \%$ agarose gel (FMC Bioproduct, Rockland, USA) containing $1 \mu \mathrm{g} / \mathrm{mL}$ of the ethidium bromide (BET) and detection by UV illumination. The gel picture was captured with an Olympus digital camera, and analysed using Digi-Doc-it software (UVP, Upland, USA).

\subsubsection{Genotyping by ERIC-PCR and REP-PCR}

The ERIC and REP (Enterobacterial Repetitive Intergenic Consensus and Repetitive Element Palindromic)-PCR, was performed using the primers ERIC-2 and Rep-2 [13]. The amplification reaction was carried out in a $25 \mu \mathrm{L}$ volume containing $2.5 \mathrm{mM}$ of $\mathrm{MgCl}_{2}, 50 \mu \mathrm{M}$ of each deoxynucleoside triphosphate, $0.3 \mu \mathrm{M}$ of the selected primers, $5 \mu \mathrm{L}$ of the DNA template and $1.5 \mathrm{U}$ of Taq DNA polymerase (Promega, Madison, Wisconsin, USA) Aliquots $(10 \mu \mathrm{L})$ of each PCR product were subjected to electrophoresis on a $1.5 \%$ agarose gel.

\subsection{Statistical Analysis}

To identify variables associated with ESBL-Kp, a risk-factor analysis was performed using a case-control study format. Demographics and hospitalisation variables of ESBL-Kp case patients were compared with patients with non-ESBL-Kp isolates. Data were entered into a database using SPSS 20.0 for Windows (SPSS Inc, Chicago, USA). The $\chi^{2}$ test and the independent samples $t$ test were used for categorical and continuous variables, respectively. A stepwise multivariate logistic regression was lead to examine the association of risk factors controlling for potential confounders. The logistic model included all variables for which a $p$-value of $<0.1$ was obtained in the multivariate analysis. A $p$-value of $<0.05$ was considered as significant.

\section{Results}

\subsection{Patient Characteristics}

Between April 2010 and March 2011, 37 of 192 (19.27\%) infected patients were included in this study and had K. pneumoniae isolates. These patients were $62.16 \%$ female and $37.84 \%$ male (F: M ratio was 1.64).Thirteen patients presented ESBL-Kp infection (35.13\%) and 24 patients had non-ESBL-Kp infections. The main epidemiological data of the infected patients by ESBL-Kp and non-ESBL-Kp isolates are shown in Table 1. The duration of hospitalisation for patients infected by ESBL-Kp isolates was $15.30( \pm 14.23)$ days, and $9.17( \pm 12.09)$ days in non-ESBL-Kp isolates $(p>0.05)$. A significant proportion of patients positive for the presence of ESBL-Kp isolates were males when compared to patients infected by non-ESBL-Kp isolates $(p=0.003)$, and the mean age of patients infected by ESBL-Kp isolates was higher than that of the patients infected by nonESBL-Kp isolates $(p=0.02)$. The wards were sorted according to the number of reported cases in each ward: the surgical ward (53.84\%), the intensive care unit (30.76\%) and the medical ward (15.38\%).

The statistical analysis showed that there was no significant difference between patients infected by ESBL-Kp and non-ESBL-Kp isolates in the infected regions. In both patients groups, the most common site of infections was the urinary tract, which yielded growth mostly in patients infected by ESBL-Kp isolates (Table 1).

Several factors, such as a previous hospitalisation, renal diseases, admission to surgical ward and urinary tract infection, were found to be significantly associated with an increased risk for acquisition of ESBL-Kp isolates (Table 1).

\subsection{Rates and Distribution of Environmental Contamination}

A total of 1000 samples were collected during the study period. Out of these cultured samples, the growth of at least one bacterial type was observed in 26.5\% ( $\mathrm{n}=265$ ), of which 115 were from equipment (monitor, aspirator, ventilator, laryngoscope, etc.) and 150 were from the care cart $(n=60)$, mattress cover $(n=40)$ and washbasin $(\mathrm{n}=50)$.

K. pneumoniae strains were identified in 99 (37.35\%) cases, and according to the contaminated ward, the medical ward (52\%) was heavily contaminated followed by the ICU, paediatric, surgery and maternity wards (Table 2). Among these isolates, 4 (4.04\%), ESBL-Kp isolates were identified from the surgery ward and the contaminated surfaces were as follow: washbasin $(n=1)$, mattress cover $(n=1)$ and care cart $(n=2)$. 
Table 1. Clinical background characteristics of patients infected and not infected with ESBL-Kp.

\begin{tabular}{|c|c|c|c|c|}
\hline Variable & $\begin{array}{c}\text { All isolates } \\
\mathrm{n}=37(\%)\end{array}$ & $\begin{array}{c}\text { ESBL-Kp isolates } \\
\mathrm{n}=13(\%)\end{array}$ & $\begin{array}{c}\text { Non-ESBL-Kpisolates } \\
n=24(\%)\end{array}$ & $p$-value \\
\hline \multicolumn{5}{|l|}{ Demographics } \\
\hline Age in years (mean $\pm \mathrm{SD}$ ) & $43 \pm 20$ & $54.53 \pm 21.36$ & $37.6 \pm 17.6$ & 0.0265 \\
\hline \multicolumn{5}{|l|}{ Gender } \\
\hline Female & $23(62.16)$ & $4(30.76)$ & 19 (79.13) & 0.005 \\
\hline Male & $14(37.83)$ & $9(69.24)$ & $5(20.87)$ & 0.003 \\
\hline Sex-ratio & 1.62 & 0.45 & 3.8 & \\
\hline \multicolumn{5}{|l|}{ Population } \\
\hline Adult & $19(51.35)$ & $4(30.76)$ & $15(62.5)$ & NS \\
\hline Elderly & $17(45.94)$ & $8(61.53)$ & $9(37.5)$ & NS \\
\hline Child & $1(2.70)$ & $1(0.6)$ & 0 & NS \\
\hline Previous hospitalisation & $19(51.35)$ & $10(76.92)$ & $9(37.5)$ & 0.02 \\
\hline Duration of hospitalisation (mean \pm SD) & $11.32 \pm 13.03$ & $15.30 \pm 14.23$ & $9.17 \pm 12.09$ & NS \\
\hline \multicolumn{5}{|l|}{ Hospital admission ward } \\
\hline Surgical & $9(24.32)$ & $7(53.84)$ & $2(8.33)$ & 0.004 \\
\hline Maternity & $14(37.83)$ & 0 & $14(58.33)$ & 0.0003 \\
\hline Medical & $6(16.21)$ & $2(15.38)$ & $4(16.66)$ & NS \\
\hline ICU & $8(21.62)$ & $4(30.76)$ & $4(16.66)$ & NS \\
\hline \multicolumn{5}{|l|}{ Infected regions } \\
\hline Respiratory samples & $4(10.81)$ & $2(15.38)$ & $2(8.33)$ & NS \\
\hline Urine samples & $22(59.45)$ & $9(69.23)$ & $13(54.16)$ & NS \\
\hline Blood samples & $2(5.40)$ & $1(7.69)$ & $1(4.16)$ & NS \\
\hline Vaginal samples & $5(13.51)$ & 0 & $5(20.83)$ & NS \\
\hline Others & $4(10.81)$ & $1(0.6)$ & $3(12.5)$ & NS \\
\hline Comorbidities & $20(54.05)$ & $12 / 13(92.30)$ & 8/24 (33.33) & NS \\
\hline Diabetes mellitus & $5(13.51)$ & 0 & $5(20.83)$ & NS \\
\hline Renal disease & $6(16.21)$ & $6(46.15)$ & 0 & 0.0007 \\
\hline Anaemia & $1(2.70)$ & $1(0.6)$ & 0 & NS \\
\hline Cerebro-vascular accident & $1(2.70)$ & $1(0.6)$ & 0 & NS \\
\hline Status epilepticus & $3(8.10)$ & $3(23.07)$ & 0 & NS \\
\hline Others & $5(13.51)$ & $2(15.38)$ & $3(12.5)$ & NS \\
\hline \multicolumn{5}{|l|}{ Reason for hospitalisation } \\
\hline Childbirth & $11(29.72)$ & 0 & $11(45.83)$ & 0.003 \\
\hline Urinary tract infection & $7(18.91)$ & $3(23.07)$ & $4(16.66)$ & NS \\
\hline Surgery & $11(29.72)$ & $7(53.84)$ & $4(16.66)$ & 0.027 \\
\hline Bronchopulmonary infections & $3(8.10)$ & $2(15.38)$ & $1(4.16)$ & NS \\
\hline Polytrauma & $3(8.10)$ & $1(7.70)$ & $2(4.16)$ & NS \\
\hline Toxaemia & $2(5.40)$ & 0 & $2(8.33)$ & NS \\
\hline \multicolumn{5}{|l|}{ Type of invasive device } \\
\hline Intubation + ventilation & $5(13.51)$ & $1(7.70)$ & $4(16.66)$ & NS \\
\hline Central venous catheter & 25 (67.56) & $5(38.46)$ & $20(83.34)$ & 0.005 \\
\hline Intubation + urinary catheter+ ventilation & $3(8.10)$ & $3(23.07)$ & 0 & 0.03 \\
\hline Urinary catheter+ central venous catheter & $4(10.81)$ & $4(30.76)$ & 0 & 0.01 \\
\hline
\end{tabular}

NS: Not significant. 
Table 2. Proportion of positive cultures according to environmental sample types and hospital units.

\begin{tabular}{ccc}
\hline & Proportion of positive cultures (\%) & Proportion of samples with K. pneumoniae present (\%) \\
\hline $\begin{array}{c}\text { Sample type } \\
\text { Surface }\end{array}$ & $(150 / 265) 56.6$ & $(60 / 99) 60.6$ \\
Equipment & $(115 / 265) 43.4$ & $(39 / 99) 39.4$ \\
Wards & & \\
ICU & $(60 / 200) 30$ & $(28 / 60) 46.66$ \\
Medical & $(50 / 200) 25$ & $(26 / 50) 52$ \\
Maternity & $(40 / 200) 20$ & $(10 / 40) 25$ \\
Paediatric & $(45 / 200) 22.5$ & $(15 / 45) 33.34$ \\
Surgery & $(70 / 200) 35$ & $(20 / 70) 28.57$ \\
Total & $(265 / 1000) 26.5$ & $(99 / 265) 37.35$ \\
\hline
\end{tabular}

\subsection{Antimicrobial Drug Susceptibility}

The antimicrobial susceptibility testing was done by the disk diffusion method of clinical and environmental isolates of K. pneumoniae.

Among 37 clinical isolates, the resistant rates for the $\beta$-lactam antibiotics amoxicillin/clavulanic acid, cephalothin, third-generation cephalosporins (ceftriaxone, ceftazidime, cefotaxime), aztreonam and cefoxitin were $81.08 \%, 81.08 \%, 35.14 \%, 35.14 \%$ and $16.22 \%$, respectively. The resistance rates for the non- $\beta$-lactam antibiotics cotrimoxazole, ciprofloxacin and gentamicin were $48.65 \%, 45.95 \%$ and $32.43 \%$, respectively.

Among the environmental $K$. pneumoniae isolates, $40.40 \%$ were resistant to amoxicillin/clavulanic acid, cephalothin and ciprofloxacin. Resistance rates to other antibiotics were as follows: $38.38 \%$ to cotrimoxazole, $28.28 \%$ to gentamicin, $13.13 \%$ to cefoxitin and $4.04 \%$ to the third-generation cephalosporins. Of the 17 ESBL-Kp isolates, including environmental and clinical isolates, all of them were multidrug-resistant and we noted that the resistance level was significantly higher than non-ESBL-Kp isolates (Table 3). The degrees of antibiotic resistance for almost all the antibiotics tested were significantly higher in the ESBL-Kp isolates compared with non-ESBL-Kp isolates (Table 3). All these isolates were susceptible to amikacin, imepenem and ertapenem, except one environmental ESBL-Kp isolate (Kp14), which was resistant to ertapenem and was positive in the modified Hodge test.

\subsection{Detection of $\beta$-Lactamases Genes}

The results of ESBL-encoding gene detection by PCR revealed that the strains studied harboured a diversity of $\beta$-lactamases, namely SHV, CTX-M and TEM (Table 4). The CTX-M ESBL-type was detected in 12 isolates (70.58\%), which all belonged to the CTX-M-1 group. Twelve (70.58\%) belonged to the SHV family and 9 (52.94\%) belonged to the TEM family. Two or more $\beta$-lactamases genes were present in 11 (64.7\%) ESBL-Kp isolates. The combination $b l a_{\mathrm{SHV}}+b l a_{\mathrm{CTX}-\mathrm{M}}$ was the most common $(4 / 17 ; 23.5 \%)$ followed by $b l a_{\mathrm{TEM}}+b l a_{\mathrm{CTX}-\mathrm{M}}$ and $b l a_{\mathrm{TEM}}+b l a_{\mathrm{SHV}}+b l a_{\mathrm{CTX}-\mathrm{M}}(3 / 17 ; 17.6 \%)$ for each and $b l a_{\mathrm{TEM}}+b l a_{\mathrm{SHV}}(2 / 17 ; 11.7 \%)$. We found that the $b l a_{\text {OXA-48 }}$ gene was present in the Kp14 carbapenem-resistant isolate which was positive in the modified Hodge test.

\subsection{Genotyping Analysis of ESBL Producers}

ERIC and REP-PCR methods were used to subtype 12 clinical and environmental ESBL-Kp isolates from during the first quarter of 2011(Table 4). The analysis differentiated the 12 isolates into four unique profiles (CK1, CK2, CK3 and CK4 clones) as shown in Figure 1(a) and Figure 1(b). The clone CK2 was represented by six isolates (Kp6, Kp7, Kp8, Kp10, Kp12 and Kp13) related to the surgical ward, but their sources were different: the isolates Kp7 and Kp10 were from the hospital environment site (Care cart) and the isolates Kp6, Kp8, Kp12 and Kp13 were isolated from infected patients (Table 4). The other three clones (CK1, CK3 and CK4) were represented by two isolates each. Clone CK1 was represented by two strains isolated in the surgical ward, one provided from the infected patient and the other from the environment site (mattress cover). The clone CK3 was 
Table 3. Antimicrobial susceptibilities of clinical and environmental ESBL-Kp strains isolated in Hassan II Hospital, Settat, Morocco.

\begin{tabular}{|c|c|c|c|c|c|c|c|c|}
\hline \multirow{3}{*}{ Antimicrobial agents } & \multicolumn{8}{|c|}{ Resistance ratios, \% (no. resistant strains) } \\
\hline & \multicolumn{3}{|c|}{ Clinical strains } & \multirow[b]{2}{*}{$p$-value } & \multicolumn{3}{|c|}{ Environmental strains } & \multirow[b]{2}{*}{$p$-value } \\
\hline & All & $\begin{array}{c}\text { ESBL } \\
(\mathrm{n}=13)\end{array}$ & $\begin{array}{c}\text { Non-ESBL } \\
(\mathrm{n}=24)\end{array}$ & & All & $\begin{array}{l}\text { ESBL } \\
(n=4)\end{array}$ & $\begin{array}{l}\text { Non-ESBL } \\
(\mathrm{n}=95)\end{array}$ & \\
\hline Amoxicillin/clavulanic acid & $81.08(30)$ & $100(13)$ & $70.83(17)$ & 0.03 & $40.40(40)$ & $100(4)$ & $37.89(36)$ & 0.02 \\
\hline Ciprofloxacin & $45.95(17)$ & $92.3(12)$ & $20.83(5)$ & $<0.0001$ & $40.40(40)$ & $100(4)$ & $37.89(36)$ & 0.02 \\
\hline Ceftriaxon & 35.14 (13) & $100(13)$ & 0 & $<0.0001$ & $4.04(4)$ & $100(4)$ & 0 & $<0.0001$ \\
\hline Ceftazidime & $35.14(13)$ & $100(13)$ & 0 & $<0.0001$ & $4.04(4)$ & $100(4)$ & 0 & $<0.0001$ \\
\hline Cefotaxime & $35.14(13)$ & $100(13)$ & 0 & $<0.0001$ & $4.04(4)$ & $100(4)$ & 0 & $<0.0001$ \\
\hline Cephalothin & $81.08(30)$ & $100(13)$ & $70.83(17)$ & 0.03 & $40.40(40)$ & $100(4)$ & $37.89(36)$ & 0.02 \\
\hline Cefoxitin & $16.22(6)$ & $38.5(5)$ & $4.16(1)$ & 0.01 & $13.13(13)$ & $25(1)$ & $12.63(12)$ & NS \\
\hline Imipenem & 0 & 0 & 0 & - & 0 & 0 & 0 & - \\
\hline Ertapenem & 0 & 0 & 0 & - & $1.01(1)$ & $25(1)$ & 0 & 0.04 \\
\hline Aztreonam & $35.14(13)$ & $100(13)$ & 0 & $<0.0001$ & 0 & 0 & 0 & - \\
\hline Amikacin & 0 & 0 & 0 & - & 0 & 0 & 0 & - \\
\hline Gentamicin & $32.43(12)$ & $84.6(11)$ & $4.16(1)$ & $<0.0001$ & $32.43(28)$ & $100(4)$ & $25.26(24)$ & 0.005 \\
\hline Cotrimoxazole & $48.65(18)$ & $61.5(8)$ & $41.66(10)$ & NS & 48.65 (38) & $50(2)$ & 37.89 (36) & NS \\
\hline
\end{tabular}

NS: Not significant.

Table 4. Characteristics of ESBL-producing K. pneumoniae detected among clinical and environmental isolates.

\begin{tabular}{|c|c|c|c|c|c|c|c|c|}
\hline \multirow{2}{*}{ Code } & \multirow{2}{*}{$\begin{array}{c}\text { Date of } \\
\text { isolation }\end{array}$} & \multirow{2}{*}{ Sex/age } & \multirow{2}{*}{ Source } & \multirow{2}{*}{ Ward } & \multirow{2}{*}{$\beta$-lactamase type } & \multicolumn{3}{|c|}{ Genotype of ESBL producer } \\
\hline & & & & & & ERIC-PCR ${ }^{*}$ profil & le REP-PCR ${ }^{*}$ profile & Clone \\
\hline Kp2 & $24 / 01 / 2011$ & $\mathrm{M} / 70$ & Urine & Surgical & CTX-M, SHV, TEM & \multirow{2}{*}{ I } & \multirow{2}{*}{1} & CK1 \\
\hline Kр4 & $01 / 02 / 2011$ & - & Mattress cover & Surgical & CTX-M, TEM & & & CK1 \\
\hline Kp6 & $18 / 01 / 2011$ & $\mathrm{M} / 36$ & Urine & Surgical & CTX-M, SHV & \multirow[b]{4}{*}{ II } & \multirow{4}{*}{2} & CK2 \\
\hline Kp7 & $01 / 02 / 2011$ & - & Care cart & Surgical & CTX-M, SHV & & & CK2 \\
\hline Kp8 & $01 / 02 / 2011$ & $\mathrm{M} / 70$ & Urine & Surgical & CTX-M & & & CK2 \\
\hline Kp10 & $01 / 02 / 2011$ & - & Care cart & Surgical & CTX-M, SHV & & & CK2 \\
\hline Kp12 & 05/01/2011 & $\mathrm{M} / 58$ & Urine & Surgical & CTX-M, SHV & & & CK2 \\
\hline Kp13 & $04 / 03 / 2011$ & $\mathrm{M} / 64$ & Pus & Surgical & SHV & & & CK2 \\
\hline Kp14 & 03/03/2011 & - & Washbasin & Surgical & CTX-M,SHV,TEM,OXA-48 & \multirow{2}{*}{ III } & \multirow{2}{*}{3} & CK3 \\
\hline Kp15 & 09/03/2011 & $\mathrm{F} / 21$ & Urine & ICU & CTX-M, SHV, TEM & & & CK3 \\
\hline Kp16 & 09/03/2011 & $\mathrm{F} / 21$ & $\begin{array}{l}\text { Protected distal } \\
\text { bronchial }\end{array}$ & ICU & SHV, TEM & \multirow{2}{*}{ VI } & \multirow{2}{*}{4} & CK4 \\
\hline Kp17 & 09/03/2011 & $\mathrm{F} / 21$ & $\begin{array}{l}\text { Protected distal } \\
\text { bronchial }\end{array}$ & ICU & SHV, TEM & & & CK4 \\
\hline Kp1 & $28 / 09 / 2010$ & $\mathrm{M} / 70$ & Urine & Surgical & CTX-M, TEM & \multicolumn{2}{|c|}{ ND } & ND \\
\hline Kp3 & $24 / 04 / 2010$ & $\mathrm{M} / 71$ & Urine & Medical & CTX-M, TEM & \multicolumn{2}{|c|}{ ND } & ND \\
\hline Kp5 & $13 / 07 / 2010$ & $\mathrm{M} / 65$ & Blood & ICU & CTX-M, TEM & \multicolumn{2}{|c|}{ ND } & ND \\
\hline Kр9 & $13 / 04 / 2010$ & $\mathrm{~F} / 74$ & Urine & Medical & SHV & \multicolumn{2}{|c|}{ ND } & ND \\
\hline Kp11 & 06/10/2010 & $\mathrm{M} / 68$ & Urine & Medical & SHV & \multicolumn{2}{|c|}{ ND } & ND \\
\hline
\end{tabular}

ND: Not determined; * The ERIC-PCR and REP-PCR profiles for K. pneumoniae isolates are shown in Figure 1. 


\section{(a)}

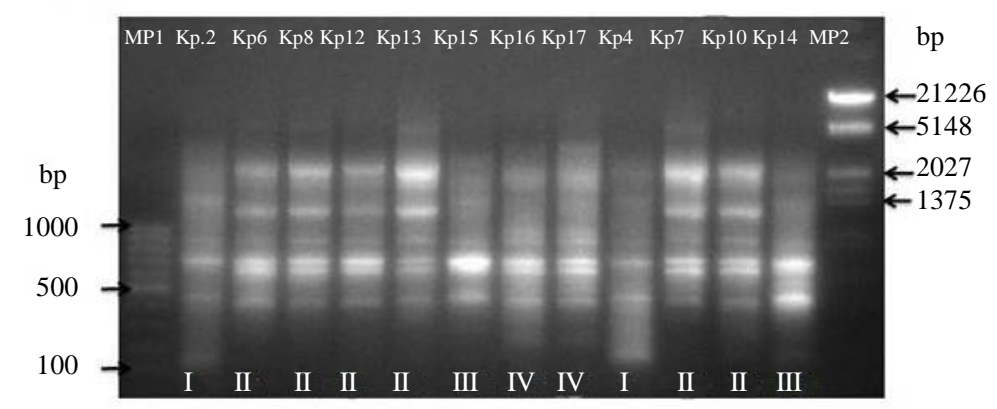

(b)

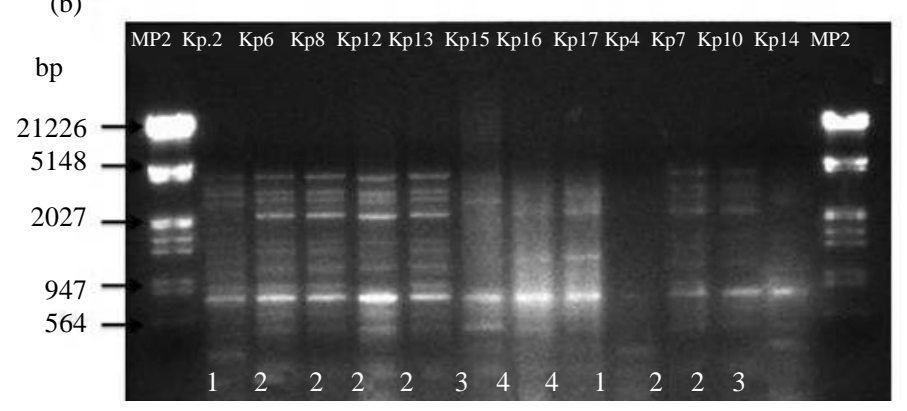

Figure 1. (a) Representative ERIC-PCR profiles for ESBL-Kpisolates. Lanes MP1: 100-bp DNA ladder, Lanes MP2: Lambda DNA was digested by EcoRI/Hind III, others lanes: isolates tested. (b) Representative REPPCR profiles for ESBL-Kpisolates. Lanes MP2: Lambda DNA was digested by EcoRI/Hind III, others lanes: isolates tested.

represented by two strains, one isolated in the surgical ward from the environment (washbasin) and the other in ICU from a hospitalised patient. The clone CK4 presented the same band between two clinical strains isolated from the ICU.

\section{Discussion}

During the last few years, ESBL-producing K. pneumoniae represented the main cause of severe infections, not only in hospitals but also in community settings. The presence of ESBL restrains the activity of a wide number of antibiotics, leading to serious therapeutic difficulties with an important impact on the outcome of the patients [1]. The prevalence of ESBL-Kp has been increasing all over the world and has varied in different countries [1]. This study provides data from a Moroccan district hospital over a one-year period. The prevalence of ESBL-Kp was 35.13\% for hospitalised patients. This rate was low compared to results reported in 2009 in Morocco, which showed a prevalence of $45 \%$ for ESBL-Kp [7]. However, this prevalence remains close to the that reported in some European and American hospitals (33\% - 35\%) [1].

The length of hospital stay mainly in ICU, mechanical ventilation, urinary catheterization, arterial catheterization, the use of a central venous catheter, and previous antibiotic treatment were found to be risk factors for the acquisition of ESBL-Kp infection along with many more [14] [15]. In this study, many risk factors for the acquisition of the ESBL-Kp infection were similar to those reported previously [14] [15]. The number of patients infected by ESBL producers receiving ventilator and catheter urinary, as well as intubation support, was higher than those infected by non-ESBL-Kp isolates. The presence of renal disease, recent surgery, male sex, and previous hospitalisation, were also found to be risk factors for the acquisition of ESBL-Kp infection.

The ERIC- and REP-PCR methods revealed four different profiles among the 12 ESBL-Kp isolates tested. The unit that was the most affected by the presence of ESBL-Kp isolates was surgery and its environmental sites (three clones). The spread of ESBL producers from one patient to another using equipment of the hospital environment has been reported many times [14]. This study confirms the serious role of the hospital environment in the transmission and spread of ESBL-producing infections in our hospital setting. Indeed, in many hospitals, the environmental contamination by ESBL-Kp is more frequent [14] [16] [17]. This could suggest that K. pneumoniae 
has a higher capacity to persist in the environment than any other species, and it is able to form biofilms that may lead to its survival during long periods in the environment [18].

In general, the resistance patterns of ESBL-Kp studied here were alike to those commonly described in other studies, the ESBL producers were resistant to different antibiotic families, including fluoroquinolones, aminoglycosides and trimethoprim/sulfamethoxazole [1] [7] [8], which contributes to the variety and the persistence of multidrug-resistant-ESBL isolates and plasmids in hospital settings, resulting from the restraint of therapeutic options. In our finding, the rate of multidrug resistance was highly alarming (100\%). Imipenem and amikacin were the most active among the antibiotics tested. It shows the real threat of this problem, particularly when we know that the increasing use of carbapenems, which have been widely used to treat serious infections with multidrug-resistant $K$. pneumoniae, has led to the appearance of carbapenem-resistant isolates via acquiring genes encoding carbapenem-hydrolysing enzymes.

In this study, one ESBL-Kp isolate was resistant to ertapenem, and was found to carry the $b l a_{\text {OXA-48 }}$ gene; the latter was the most frequent gene reported in North African and Mediterranean countries [19] [20]. Recently, a nosocomial dissemination of OXA-48-producing K. pneumoniae isolates has been reported in Moroccan hospitals [21]-[23].

The screening of blagenes detected a diversity of $\beta$-lactamases, and the CTX-M type was the most common ESBL in our hospital. The predominance of CTX-M-15 is now common in Morocco, as in other countries, as a result of worldwide expansion [4] [5]. These high rates of CTX-M among total ESBL enzymes are most probably associated with the high mobilisation of the encoding genes, as it has been found that $b l a_{\text {CTX-M }}$ genes are mobilised to plasmids more frequently than other class A $\beta$-lactamases [24]. The genes encodedby the SHV family were detected in $70.58 \%$ of the isolates, and most enzymes of this group are ESBL, except for SHV-1 and SHV-11 [25]. However, the enzymes of this group cannot be confirmed to be ESBL-encoding genes, particularly in strains presenting more than one bla gene. On the other hand, the $b l a_{\mathrm{SHV}-12}$ and $b l a_{\mathrm{SHV}-27}$ genes were also detected in K. pneumoniae from Moroccan hospitals [21] [26]. The bla $a_{\text {TEM }}$ gene was identified in $47.05 \%$ of isolates, usually in association with SHV and/or CTX-M genes. Therefore, it was not possible to determine whether they were responsible for the ESBL phenotype; they could be non-ESBL-encoding genes associated with another gene expressing the extended-spectrum phenotype [25]. Conversely, the co-production of various ESBL by a unique strain has been a common result in many studies [5] [27] [28].

\section{Conclusion}

In conclusion, several clones of ESBL-Kp are widespread and are circulating both in the hospital environment and in patients. These strains harbour two or more $\beta$-lactamases genes along with the emergence of $b l a_{\text {OXA-48 }}$ gene. A variety of risk factors associated with the occurrence of ESBL-Kp were detected. In summary, there is an urgent need to increase hygiene measures in order to limit the spread of these multidrug-resistant organisms in the hospital.

\section{References}

[1] Sader, H.S., Farrell, D.J., Flamm, R.K. and Jones, R.N. (2014) Antimicrobial Susceptibility of Gram-Negative Organisms Isolated from Patients Hospitalized in Intensive Care Units in United States and European Hospitals (2009-2011). Diagnostic Microbiology and Infectious Disease, 78, 443-448. http://dx.doi.org/10.1016/j.diagmicrobio.2013.11.025

[2] Vodovar, D., et al. (2013) Enterobacteriaceae Producing Extended Spectrum Beta-Lactamase: Epidemiology, Risk Factors, and Prevention. La Revue de Médecine Interne, 34, 687-693. http://dx.doi.org/10.1016/j.revmed.2012.10.365

[3] Philippon, A. (2013) Les bêta-lactamases à spectre élargi ou étendu (BLSE). Immuno-analyse \& Biologie Spécialisée, 28, 287-296. http://dx.doi.org/10.1016/j.immbio.2013.04.006

[4] Ruppé, E. (2010) Épidémiologie des bêta-lactamases à spectre élargi: l’avènement des CTX-M. Antibiotiques, 12, 3-16. http://dx.doi.org/10.1016/j.antib.2010.01.003

[5] Barguigua, A., et al. (2013) Prevalence and Genotypic Analysis of Plasmid-Mediated Beta-Lactamases among Urinary: Klebsiella pneumoniae Isolates in Moroccan Community. Journal of Antibiotics, 66, 11-16. http://dx.doi.org/10.1038/ja.2012.91

[6] Nedjai, S., et al. (2012) Prevalence and Characterization of Extended Spectrum Beta-Lactamases in KlebsiellaEnterobacter-Serratia Group Bacteria, in Algeria. Médecine et Maladies Infectieuses, 42, 20-29. http://dx.doi.org/10.1016/j.medmal.2011.10.001

[7] Lahlou Amine, I., Chegri, M. and L’Kassmi, H. (2009) Épidémiologie et résistance aux antibiotiques des entéro- 
bactéries isolées d’infections urinaires à l’hôpital militaire Moulay-Ismail de Meknès. Antibiotiques, 11, 90-96. http://dx.doi.org/10.1016/j.antib.2008.10.004

[8] El Bouamri, M.C., et al. (2014) Recent Evolution of the Epidemiological Profile of Extended-Spectrum BetaLactamase Producing Uropathogenic Enterobacteria in Marrakech, Morocco. Progrès en Urologie, 24, 451-455. http://dx.doi.org/10.1016/j.purol.2013.11.010

[9] Soraas, A., et al. (2013) Risk Factors for Community-Acquired Urinary Tract Infections Caused by ESBL-Producing Enterobacteriaceae-A Case-Control Study in a Low Prevalence Country. PLoS One, 8, e69581. http://dx.doi.org/10.1371/journal.pone.0069581

[10] CLSI (2011) Clinical and Laboratory Standards Institute, Performance Standards for Antimicrobial Susceptibility Testing: Twenty-First Informational Supplement M100-S21. CLSI, Wayne, PA.

[11] Garrec, H., et al. (2011) Comparison of Nine Phenotypic Methods for Detection of Extended-Spectrum Beta-Lactamase Production by Enterobacteriaceae. Journal of Clinical Microbiology, 49, 1048-1057. http://dx.doi.org/10.1128/JCM.02130-10

[12] Barguigua, A., et al. (2012) Emergence of Carbapenem-Resistant Enterobacteriaceae Isolates in the Moroccan Community. Diagnostic Microbiology \& Infectious Diseases, 73, 290-291. http://dx.doi.org/10.1016/j.diagmicrobio.2012.03.011

[13] Lim, K.T., et al. (2009) Characterization of Multidrug Resistant ESBL-Producing Escherichia coli Isolates from Hospitals in Malaysia. J Biomed Biotechnol., 2009(10): p. 165637.

[14] van’t Veen, A., et al. (2005) Outbreak of Infection with a Multiresistant Klebsiella pneumoniae Strain Associated with Contaminated Roll Boards In Operating Rooms. J Clin Microbiol., 43, 4961-4967. http://dx.doi.org/10.1128/JCM.43.10.4961-4967.2005

[15] Bisson, G., et al. (2002) Extended-Spectrum Beta-Lactamase-Producing Escherichia coli and Klebsiella Species: Risk Factors for Colonization and Impact of Antimicrobial Formulary Interventions on Colonization Prevalence. Infect Control Hosp Epidemiol., 23, 254-260. http://dx.doi.org/10.1086/502045

[16] Arabaci, F., Oldacay, M. and Berber, D. (2009) Evaluation of ESBL Positivity Rates for Escherichia coli and Klebsiella pneumoniae Strains with the Sensititre ESBL Antimicrobic Susceptibility Plates in a Public Hospital, Turkey. Journal of Medical Sciences, 9, 4. http://dx.doi.org/10.3923/jms.2009.103.107

[17] Guet-Revillet, H., et al. (2012) Environmental Contamination with Extended-Spectrum $\beta$-Lactamases: Is There Any Difference between Escherichia coli and Klebsiella spp? American Journal of Infection Control, 40, 845-848. http://dx.doi.org/10.1016/j.ajic.2011.10.007

[18] Jones, K. and Bradshaw, S.B. (1997) Synergism in Biofilm Formation between Salmonella enteritidis and a NitrogenFixing Strain of Klebsiella pneumoniae. Journal of Applied Microbiology, 82, 663-668. http://dx.doi.org/10.1111/j.1365-2672.1997.tb03600.x

[19] Poirel, L., Potron, A. and Nordmann, P. (2012) OXA-48-Like Carbapenemases: The Phantom Menace. Journal of Antimicrobial Chemotherapy, 67, 1597-1606. http://dx.doi.org/10.1093/jac/dks121

[20] Nordmann, P., Naas, T. and Poirel, L. (2011) Global Spread of Carbapenemase-Producing Enterobacteriaceae. Emerging Infectious Diseases, 17, 1791-1798. http://dx.doi.org/10.3201/eid1710.110655

[21] Hays, C., et al. (2012) Nosocomial Occurrence of OXA-48-Producing Enterobacterial Isolates in a Moroccan Hospital. International Journal of Antimicrobial Agents, 39, 545-547. http://dx.doi.org/10.1016/j.ijantimicag.2012.03.002

[22] Poirel, L., et al. (2011) Emergence of NDM-1-Producing Klebsiella pneumoniae in Morocco. Journal of Antimicrobial Chemotherapy, 66, 2781-2783. http://dx.doi.org/10.1093/jac/dkr384

[23] Poirel, L., et al. (2011) Cross-Border Transmission of OXA-48-Producing Enterobacter cloacae from Morocco to France. Journal of Antimicrobial Chemotherapy, 66, 1181-1182. http://dx.doi.org/10.1093/jac/dkr023

[24] Barlow, M., et al. (2008) High Rate of Mobilization for blaCTX-Ms. Emerging Infectious Diseases, 14, 423-428. http://dx.doi.org/10.3201/eid1403.070405

[25] Livermore, D.M. (2008) Defining an Extended-Spectrum Beta-Lactamase. Clinical Microbiology and Infections, 14, 310. http://dx.doi.org/10.1111/j.1469-0691.2007.01857.x

[26] Bouchakour, M., et al. (2010) Plasmid-Mediated Quinolone Resistance in Expanded Spectrum Beta Lactamase Producing Enterobacteriaceae in Morocco. Journal of Infection in Developing Countries, 4, 779-803.

[27] Li, C.R., Li, Y. and Zhang, P.A. (2003) Dissemination and Spread of CTX-M Extended-Spectrum Beta-Lactamases among Clinical Isolates of Klebsiella pneumoniae in Central China. International Journal of Antimicrobial Agents, 22, 521-525. http://dx.doi.org/10.1016/S0924-8579(03)00157-2

[28] Paterson, D.L., et al. (2000) Epidemiology of Ciprofloxacin Resistance and Its Relationship to Extended-Spectrum Beta-Lactamase Productionin Klebsiella pneumoniae Isolates Causing Bacteremia. Clinical Infectious Diseases, 30, 473-478. http://dx.doi.org/10.1086/313719 